\title{
LIVING AND QUATERNARY OSTRACODA FROM THE EASTERN ADRIATIC SEA: BIOCOENOSES, THANATOCOENOSES OR PALAEOTHANATOCOENOSES?
}

\author{
HeNNing UfFenORde ${ }^{1 *}$ \\ ${ }^{1}$ Geoscience Centre, GZG, Museum, Collections \& Geopark, Göttingen University, Göttingen, \\ Germany (e-mail: huffeno@gwdg.de)
}

Uffenorde, H.: Living and Quaternary Ostracoda from the Eastern Adriatic Sea: Biocoenoses, thanatocoenoses or palaeothanatocoenoses? Nat. Croat., Vol. 25, No. 1, 73-86, 2016, Zagreb.

Many new ostracod species from the Northern Adriatic Sea are based on descriptions of empty valves that were nevertheless interpreted as being parts of recent marine habitats and listed in several zoological checklists and databases. Sedimentary-geological studies contradict this interpretation. Ostracods from 2 cores taken off the Istrian Coast prove the existence of a relatively uniform subsurface layer with a rich death assemblage from a palaeoenvironmental depth zone prior or equal to the sea-level that existed 7-5 ka BP, related to the postglacial sea-level change. This layer was covered by patches with extremely different ostracod bio-, thanato- or palaeothanatocoenoses, depending on storm re-deposition, lag deposition or bioturbation. In terms of sequence stratigraphy, the Maximum Flooding Surface and a condensed Highstand Systems Tract are still forming the bottom areas more than $5 \mathrm{~km}$ off the Istrian Coast.

Key words: Ostracoda, Crustacea, biocoenosis, thanatocoenosis, palaeothanatocoenosis, Quaternary, type locality, Adriatic Sea

Uffenorde, H.: Recentni i kvartarni ostrakodi iz istočnog Jadrana: biocenoze, tanatocenoze ili paleotanatocenoze? Nat. Croat., Vol. 25, No. 1, 73-86, 2016, Zagreb.

Mnogo novih vrsta ostrakoda iz sjevernog Jadrana opisano je na temelju praznih ljuštura. Bez obzira na to, vrste su interpretirane kao dio recentnih morskih staništa i navedene u nekoliko zooloških popisa vrsta i baza podataka. Sediment iz geoloških istraživanja proturječi ovakvoj interpretaciji. Ostrakodi iz dviju jezgri izvađenih blizu obale Istre dokazuju postojanje relativno jednoličnog potpovršinskog sloja s bogatom zajednicom mrtvih organizama, iz paleookolišne dubinske zone ispod ili u razini razine mora, koja je nastala prije 7-5 ka BP zbog postglacijalne promjene razine mora. Taj sloj bio je pokriven dijelovima izrazito raznolikih ostrakodnih bio-, tanato- ili paleotanatocenoza, ovisno o njihovoj preraspodjeli zbog djelovanja oluja, zaostalih taloženja ili bioturbaciji. Što se tiče sekvencijalne stratigrafije, Površina maksimalnog preplavljivanja (MFS) i kondenzirani Trakt visoke razine (HST) još uvijek se stvaraju na dnima udaljenim više od $5 \mathrm{~km}$ od obale Istre.

Ključne riječi: Ostracoda, Crustacea, biocenoza, tanatocenoza, paleotanatocenoza, kvartar, tipski lokalitet, Jadransko more

\section{INTRODUCTION}

In the years between 1960 and 1980, marine ostracods from the present-day sea floor of the Adriatic Sea were examined mainly from the Italian coast and the international

* extended version of a presentation held at the EOM 8 in Tartu, Estonia (UfFenorde, 2015) 
offshore areas from (Ascoli, 1965; MAsoli, 1968, 1969; BonAduce et al., 1976; Ciliberto \& Pugliese, 1980).

A German research group under the guidance of Prof. Meischner (Göttingen) undertook sedimentological and geochemical studies (Fütterer, 1969; PAul, 1970; Meischner, 1973; Fütterer \& PAUL, 1976) as well as investigations of the taxonomy, ecology and seasonality of foraminifers and ostracods along the Istrian coast and Limski kanal $\mathrm{N}$ of Rovinj (von Daniels, 1970; Uffenorde, 1972, 1975). Palynological data were added by BEUg (1977). For further descriptions of the ecological conditions, for example with sponge habitats in the area off the Istrian coast and Limski kanal, see KLÖPpEL et al. (2011).

The Italian scientists mentioned above, as well as SокAс̌ (1975) and SокAс̌ \& HajEKTADESSE (1993) from the Croatian side, predominantly used the Van Veen grab sampler, whereas the equipment used by the Göttingen group was a high momentum gravity corer (core A18, Meischner \& Rumohr, 1974), a box corer (core PO-1, "Kastenlot Kiel" FüTtERER, 1969, for the position of both cores see text-fig. 1) and an improved Krumm bottom sampler (Limski kanal stations: von DANIELs et al., 1970). This equipment and the procedure for bringing the samples onboard seem to be of importance for the integrity of the microfaunal sample composition, whether they belong to a biocoenosis (living assemblage), a thanatocoenosis (death assemblage) or a palaeothanatocoenosis (death assemblage from a different climatic provinces = palaeothanatocoenosis I of BremAN (1976) or from a different palaeoenvironmental depth zone due to the postglacial sealevel change $=$ palaeothanatocoenosis II of BREMAN (1976)).

In connection with sampling cruises by Trotтi (1969), Bonaduce et al. (1976) completed a huge study of Ostracoda, describing 46 new species and 2 new genera. Although all species are listed in Tab. 1 together with the latitudes and longitudes of their type localities, only the northernmost traverse from the Po delta towards the Mirna river/ Istrian coast with the type localities of 6 new species from $34 \mathrm{~m}$ water depth or less are of special interest in the context of this publication (stations 2 to 5 of BonADuce et al. (1976); refigured with Cyprideis torosa findings by BonAduce et al. (2004)). These taxa of BonAduce et al. (1976) are Loxoconcha geometrica, Semicytherura rarecostata, Callistocythere folliculosa, Callistocythere gilva, Loxoconcha exagona - together with the revised homonymous species Leptocythere bituberculata (= Leptocythere istriana Kempf, 2011).

All these ostracod taxa are based on descriptions of empty valves and therefore per se belong to the present-day thanatocoenosis. Nevertheless, they were interpreted as being part of the living inventory of the Adriatic Sea (MASOLI, 1968; BonAducE et al., 1976; BONADUCE et al., 2004). Since then, this attribution has been repeatedly published in summarizing checklists (Aiello et al., 1995; Montenegro et al., 1998; Aiello \& BARRA, 2010). Modern databases like "World Register of Marine Species" (WoRMS)/“World Ostracoda Database", "Encyclopedia of Life" (eol), North Atlantic Register for Marine Species (NARMS), "European Register of Marine Species" (ERMS)/MarBEF Data System" (marbef) and other zoological databases have followed this interpretation.

The attribution of these ostracods to the living fauna has to be questioned in view of sedimentary-geological studies which can be traced back to van STRAATEN (1970) and Fütterer \& Paul (1976). Van StraAten (1970) emphasized that recent sands are restricted predominantly to a narrow coastal zone, and Pleistocene to Holocene residual sands were distributed widely on the shelf during the late glacial to post-glacial sealevel rise. BREMAN (1976) studied about 300 samples from the 360 stations visited by Van Straaten in 1962. In his tab. III d, he assigned the bottom sediments of the stations 134 to 138, which are relevant in this context, to the Pleistocene. In contrast to Bonaduce et al. 


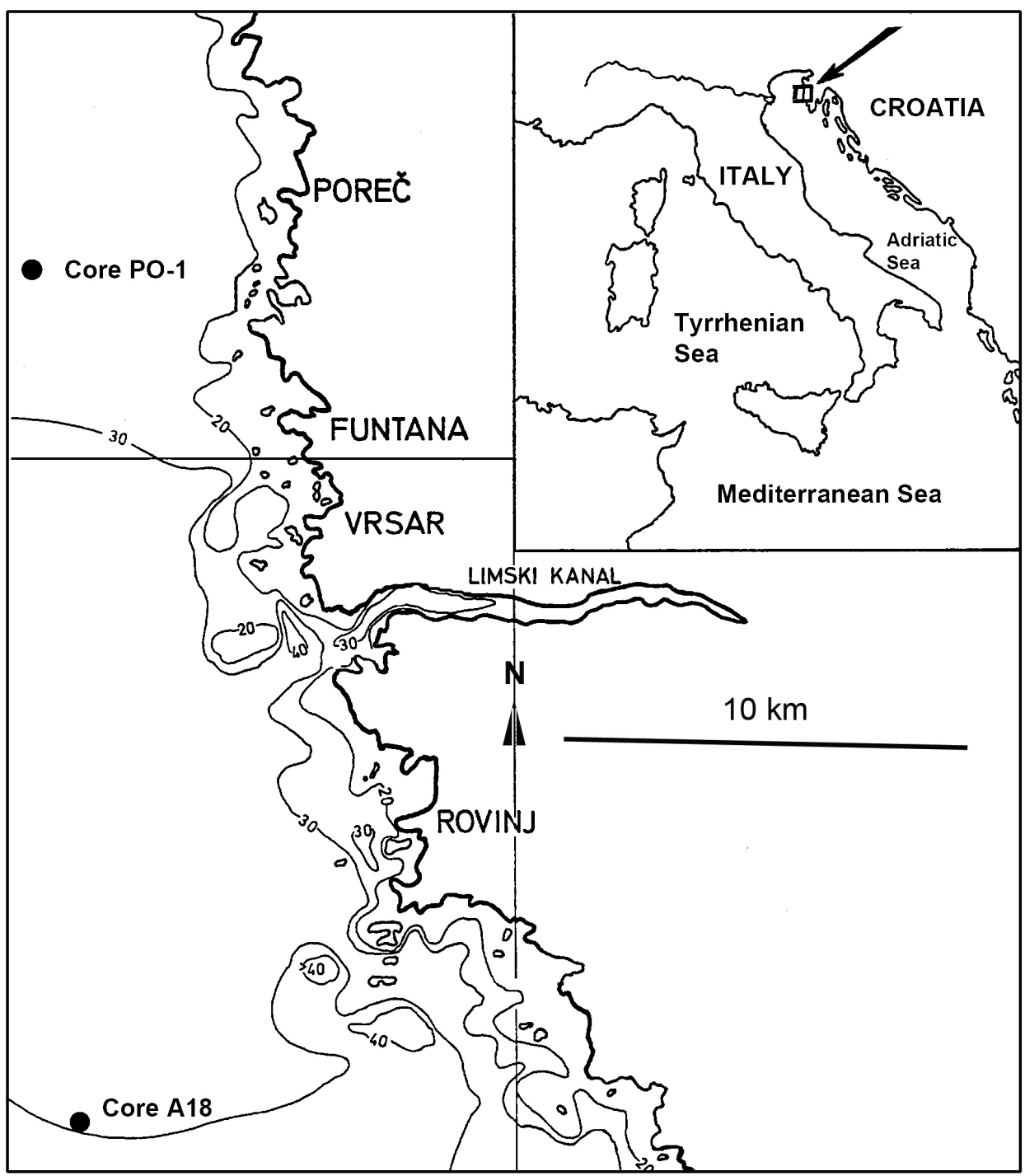

Fig. 1. Sketch map of the northeastern Adriatic Sea with the cores PO-1 and A18 offshore Istria, Croatia (modified, after FüTTERER, 1969).

(2004), BREMAN (1976) found only in one station (station 136) of this area Cyprideis torosa (10\% of the counted valves), which clearly indicated to him that the sediment sample belongs to a palaeothanatocoenosis.

On the Istrian side of the Adriatic Sea, recent sedimentation is mainly confined to a 2 to $4 \mathrm{~km}$ wide zone (the "sediment zone I" of Fütterer \& PAul (1976)). The underlying karst morphology causes a very patchy distribution of reworked "terra rossa" and skeletal debris. Further offshore, "sediment zone II" is a $2 \mathrm{~km}$ wide strip, which is still poorly sorted and free of Montmorillonite. "Sediment zone III", 5 to $15 \mathrm{~km}$ off the Is- 
Tab. 1. Type localities of the new species described by Bonaduce et al. (1976). Latitude and longitude data after TRотті (1969), stations located in combination with hydrographical data. Digital version: OGA National Oceanographic Data Centre, Trieste.

\begin{tabular}{|c|c|c|c|c|}
\hline $\begin{array}{c}\text { Stations Bonaduce, } \\
\text { Ciampo \& Masoli } \\
\text { [BCM], 1976 [No.] }\end{array}$ & Type locality of taxon & Latitude & Longitude & $\begin{array}{c}\text { Depth } \\
\text { [m below } \\
\text { water surface] }\end{array}$ \\
\hline 2 & Loxoconcha geometrica BCM, 1976 & $45^{\circ} 3.00^{\prime} \mathrm{N}$ & $12^{\circ} 48.00^{\prime} \mathrm{E}$ & 36 \\
\hline 3 & Semicytherura rarecostata BCM, 1976 & $45^{\circ} 6.96^{\prime} \mathrm{N}$ & $13^{\circ} 1.98^{\prime} \mathrm{E}$ & 32 \\
\hline 4 & Callistocythere folliculosa BCM, 1976 & $45^{\circ} 9.96^{\prime} \mathrm{N}$ & $13^{\circ} 12.96^{\prime} \mathrm{E}$ & 34 \\
\hline 4 & Callistocythere gilva BCM, 1976 & $45^{\circ} 9.96^{\prime} \mathrm{N}$ & $13^{\circ} 12.96^{\prime} \mathrm{E}$ & 34 \\
\hline 5 & $\begin{array}{l}\text { Leptocythere istriana Kempf, } 2011 \\
\text { (= L.bituberculata BCM, 1976), } \\
\text { Loxoconcha exagona BCM, 1976 }\end{array}$ & $45^{\circ} 13.98^{\prime} \mathrm{N}$ & $13^{\circ} 21.96^{\prime} \mathrm{E}$ & 27 \\
\hline 19 & Pontocypris acuta BCM, 1976 & $43^{\circ} 57.00^{\prime} \mathrm{N}$ & $14^{\circ} 1.98^{\prime} \mathrm{E}$ & 71 \\
\hline 20 & Microcythere vitrea BCM, 1976 & $44^{\circ} 3.96^{\prime} \mathrm{N}$ & $14^{\circ} 12.96^{\prime} \mathrm{E}$ & 71 \\
\hline 20 & Cytheromorpha nana BCM, 1976 & $44^{\circ} 3.96^{\prime} \mathrm{N}$ & $14^{\circ} 12.96^{\prime} \mathrm{E}$ & 71 \\
\hline 30 & Cytheropteron hadriaticum BCM, 1976 & $43^{\circ} 28.98^{\prime} \mathrm{N}$ & $15^{\circ} 7.98^{\prime} \mathrm{E}$ & 125 \\
\hline 30 & $\begin{array}{l}\text { Loxoconcha bonaciamma Kempf, } 2011 \\
(=\text { L. concentrica BCM, 1976) }\end{array}$ & $43^{\circ} 28.98^{\prime} \mathrm{N}$ & $15^{\circ} 7.98^{\prime} \mathrm{E}$ & 125 \\
\hline 31 & Semicytherura stilifera BCM, 1976 & $43^{\circ} 34.98^{\prime} \mathrm{N}$ & $15^{\circ} 16.98^{\prime} \mathrm{E}$ & 117 \\
\hline 41 & ?Cytheropteron infelix BCM, 1976 & $42^{\circ} 18.96^{\prime} \mathrm{N}$ & $14^{\circ} 39.00^{\prime} \mathrm{E}$ & 71 \\
\hline 44 & Argilloecia micra BCM, 1976 & $42^{\circ} 37.98^{\prime} \mathrm{N}$ & $15^{\circ} 4.98^{\prime} \mathrm{E}$ & 180 \\
\hline 44 & Cytheropteron garganicum BCM, 1976 & $42^{\circ} 37.98^{\prime} \mathrm{N}$ & $15^{\circ} 4.98^{\prime} \mathrm{E}$ & 180 \\
\hline 51 & Callistocythere vexata BCM, 1976 & $43^{\circ} 13.98^{\prime} \mathrm{N}$ & $15^{\circ} 57.96^{\prime} \mathrm{E}$ & 113 \\
\hline 69 & Buntonia textilis BCM, 1976 & $42^{\circ} 9.00^{\prime} \mathrm{N}$ & $16^{\circ} 27.00^{\prime} \mathrm{E}$ & 133 \\
\hline 69 & Paracytherois mediterranea BCM, 1976 & $42^{\circ} 9.00^{\prime} \mathrm{N}$ & $16^{\circ} 27.00^{\prime} \mathrm{E}$ & 133 \\
\hline 70 & Polycope truncatula BCM, 1976 & $42^{\circ} 13.98^{\prime} \mathrm{N}$ & $16^{\circ} 34.98^{\prime} \mathrm{E}$ & 170 \\
\hline 70 & Rectobuntonia miranda BCM, 1976 & $42^{\circ} 13.98^{\prime} \mathrm{N}$ & $16^{\circ} 34.98^{\prime} \mathrm{E}$ & 170 \\
\hline 70 & Eucythere pubera BCM, 1976 & $42^{\circ} 13.98^{\prime} \mathrm{N}$ & $16^{\circ} 34.98^{\prime} \mathrm{E}$ & 170 \\
\hline 70 & Parakrithe dimorpha $\mathrm{BCM}, 1976$ & $42^{\circ} 13.98^{\prime} \mathrm{N}$ & $16^{\circ} 34.98^{\prime} \mathrm{E}$ & 170 \\
\hline 70 & Typhlocythere ruggierii BCM, 1976 & $42^{\circ} 13.98^{\prime} \mathrm{N}$ & $16^{\circ} 34.98^{\prime} \mathrm{E}$ & 170 \\
\hline 70 & „Bythoceratina“ reticulata BCM, 1976 & $42^{\circ} 13.98^{\prime} \mathrm{N}$ & $16^{\circ} 34.98^{\prime} \mathrm{E}$ & 170 \\
\hline 70 & Monoceratina oblita BCM, 1976 & $42^{\circ} 13.98^{\prime} \mathrm{N}$ & $16^{\circ} 34.98^{\prime} \mathrm{E}$ & 170 \\
\hline 70 & Loxoconchidea minima BCM, 1976 & $42^{\circ} 13.98^{\prime} \mathrm{N}$ & $16^{\circ} 34.98^{\prime} \mathrm{E}$ & 170 \\
\hline 70 & Cythropteron sulcatum BCM, 1976 & $42^{\circ} 13.98^{\prime} \mathrm{N}$ & $16^{\circ} 34.98^{\prime} \mathrm{E}$ & 170 \\
\hline 70 & ?Cytheropteron tetrapteron BCM, 1976 & $42^{\circ} 13.98^{\prime} \mathrm{N}$ & $16^{\circ} 34.98^{\prime} \mathrm{E}$ & 170 \\
\hline 71 & Polycope parareticulata BCM, 1976 & $42^{\circ} 21.00^{\prime} \mathrm{N}$ & $16^{\circ} 45.96^{\prime} \mathrm{E}$ & 210 \\
\hline 71 & Polycopsis quadridentata BCM, 1976 & $42^{\circ} 21.00^{\prime} \mathrm{N}$ & $16^{\circ} 45.96^{\prime} \mathrm{E}$ & 210 \\
\hline 71 & Cytherella alvearium BCM, 1976 & $42^{\circ} 21.00^{\prime} \mathrm{N}$ & $16^{\circ} 45.96^{\prime} \mathrm{E}$ & 210 \\
\hline 71 & Cytheropteron zinzulusae BCM, 1976 & $42^{\circ} 21.00^{\prime} \mathrm{N}$ & $16^{\circ} 45.96^{\prime} \mathrm{E}$ & 210 \\
\hline 72 & Pedicythere phryne BCM, 1976 & $42^{\circ} 27.96^{\prime} \mathrm{N}$ & $16^{\circ} 57.96^{\prime} \mathrm{E}$ & 212 \\
\hline
\end{tabular}




\begin{tabular}{|c|l|c|c|c|}
\hline 74 & Polycope tholiformis BCM, 1976 & $42^{\circ} 30.96^{\prime} \mathrm{N}$ & $17^{\circ} 1.98^{\prime} \mathrm{E}$ & 194 \\
\hline 74 & Argilloecia robusta BCM, 1976 & $42^{\circ} 30.96^{\prime} \mathrm{N}$ & $17^{\circ} 1.98^{\prime} \mathrm{E}$ & 194 \\
\hline 78 & Cytheropteron monoceros BCM, 1976 & $43^{\circ} 31.98^{\prime} \mathrm{N}$ & $14^{\circ} 18.00^{\prime} \mathrm{E}$ & 85 \\
\hline 78 & Cytheropteron venustum $\mathrm{BCM}, 1976$ & $43^{\circ} 31.98^{\prime} \mathrm{N}$ & $14^{\circ} 18.00^{\prime} \mathrm{E}$ & 85 \\
\hline 81 & Semicytherura robusta BCM, 1976 & $43^{\circ} 42.96^{\prime} \mathrm{N}$ & $14^{\circ} 34.98^{\prime} \mathrm{E}$ & 79 \\
\hline 100 & "Pedicythere“ tessellata BCM, 1976 & \multicolumn{2}{|c|}{ unknown } & 110 \\
\hline
\end{tabular}

trian Coast, is uniformly better sorted (grain size maxima about $160 \mu$ ), although containing 70 to $80 \%$ carbonate, like zone II. It belongs to the Montmorillonite clay mineral province, which is predominant in the Northern Adriatic Sea. The cores PO-1 and A18, the ostracods of which are discussed below, originate from zone III.

\section{RESULTS}

\section{Ostracoda from core PO-1, WSW of Poreč/Istria}

The 100 cm long core PO-1 (Fütterer, 1969; Fütterer \& PAul, 1976; Beug, 1977) (position: $45^{\circ} 23^{\prime} 37^{\prime \prime} \mathrm{N} 13^{\circ} 00^{\prime} 31^{\prime \prime} \mathrm{E}$, water depth: $28 \mathrm{~m}$; Fig. 1) shows a subdivision into 4 units:

Unit D $\quad 0-5 \mathrm{~cm}$ : fine sand, few large mollusk shells

Unit C 5-20 cm: shell layer (Ostrea edulis, Arca noae, Pecten sp., Cerithium rupestre, Murex sp., det. Fütterer \& PAul (1976)

Unit B 20-50 cm: transition to sandy silt

Unit A 50-100 cm: clayey silt, minor sand content, numerous vertical and horizontal burrows, mollusk shells rare.

Core PO- 1 contains a relatively uniform ostracod assemblage, especially from $20 \mathrm{~cm}$ down to base (Tab. 2). Only the surface $(0-3 \mathrm{~cm})$ sample did not contain any Cyprideis torosa, while sample 5-10 cm and all deeper samples clearly indicate a palaeothanatocoenosis II. UfFenorde (in FütTERER \& PAul, 1976: p. 16) found abundant Cyprideis torosa in sample 95-100 cm, assigning the source to "a neighbouring brackish water mass (lagoon?) or a wide zone of brackish water with a seaward overflow". BEUG (1977) added that this finding is a further indication for an onlapping Adriatic Sea at about 7000-8000 a BP. FütTERER \& PAUL (1976) came to the conclusion that units A and B of the core might have their origin in a "shallow-water environment near to the coast ... comparable with the present coastal zone between Grado and Venice". Recently, Dinelli et al. (2012) described a similar assemblage from the southern Po Plain as a lagoon facies association within a late glacial-early Holocene transgressive back-barrier succession. A sample from this lagoon facies association could be dated with the ${ }^{14} \mathrm{C}$ method as being from $7627 \pm 172$ cal. years BP (Dinelli et al. (2012: text-fig. 3).

Near the center of the Po Delta, this typical lagoon facies association has not been found, due to a different palaeoenvironmental development (Rossi, 2009). Transgressive barrier sands are overlain by marine inner shelf clays (ca. 5.,8 ka BP and younger), containing an ostracode assemblage dominated by Pontocythere turbida (G. W. MüLLER, 1894) and Semicytherura acuticostata (SARS, 1866) (assemblage 2 Rossi, 2009).

Cyprideis torosa is generally smooth, and adult nodose morphotypes occur very rarely, while the brackish-marine species Leptocythere bacescoi and Loxoconcha stellifera are confined to the lower part of the core. 
Tab. 2. Ostracod distribution, ${ }^{14} \mathrm{C}$ and palynological data of core PO-1, WSW of Poreč/Istria. Light grey arrows towards left: shallow-marine species. Dark grey arrows towards right: brackish-lagoonal species.

\begin{tabular}{|c|c|c|c|c|c|c|c|c|c|c|c|c|}
\hline $\begin{array}{l}\text { Core PO-1 } \\
\text { Core No. }\end{array}$ & 1 & 3 & 5 & 7 & 9 & 11 & 13 & 15 & 16 & 17 & 19 & 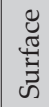 \\
\hline Core depth $[\mathrm{cm}]$ & $\begin{array}{l}8 \\
\stackrel{1}{1} \\
\text { ถ⿱ }\end{array}$ & $\begin{array}{l}8 \\
8 \\
1 \\
\infty\end{array}$ & $\begin{array}{l}\infty \\
\text { o } \\
\text { 占 }\end{array}$ & $\begin{array}{l}\text { R } \\
\text { ํํㅇ }\end{array}$ & $\begin{array}{l}8 \\
8 \\
1 \\
10\end{array}$ & $\begin{array}{l}\text { டி } \\
\text { டி } \\
\text { 字 }\end{array}$ & $\begin{array}{l}\text { P } \\
1 \\
\text { 岁 }\end{array}$ & 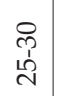 & 菅 & 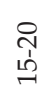 & 올 & $\begin{array}{l}\text { mo } \\
\vdots \\
1\end{array}$ \\
\hline \multicolumn{13}{|l|}{ Ostracod taxa } \\
\hline Aurila convexa (Baird, 1850) & $x$ & & $x$ & $x$ & $\mathrm{X}$ & $x$ & 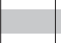 & $\mathrm{X}$ & 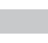 & 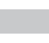 & $x$ & $\mathrm{X}$ \\
\hline Aurila ithacae Uliczny, 1969 & $x$ & & & $x$ & & & $\mathrm{X}$ & & $x$ & $x$ & & \\
\hline Neonesidea mediterranea (G. W. Müller, 1894) & & & & & & & & & $\mathrm{X}$ & & & \\
\hline Callistocythere adriatica Masoli, 1968 & $x$ & $?$ & $x$ & $x$ & $x$ & $x$ & $\mathrm{X}$ & $\mathrm{X}$ & $x$ & $x$ & $x$ & $\mathrm{X}$ \\
\hline Callistocythere littoralis (G. W. Müller, 1894) & & & & $\mathrm{X}$ & $\mathrm{X}$ & $x$ & & & & & & $x$ \\
\hline Carinocythereis princeps (Terquem, 1878) & $x$ & & & & & & & & & & & \\
\hline Carinocythereis whitei (Baird, 1850) & & & $x$ & $X$ & $X$ & $x$ & $\mathrm{X}$ & $\mathrm{X}$ & . & $x$ & $x$ & $\mathrm{X}$ \\
\hline \multicolumn{13}{|l|}{ Cyprideis torosa (Jones, 1850) } \\
\hline $\begin{array}{l}\text { Cyprideis torosa (Jones, 1850) (nodose } \\
\text { morphotype) }\end{array}$ & & $x$ & & & & & & $x$ & & & & \\
\hline Cytheretta subradiosa (Roemer, 1838) & $x$ & & & & $x$ & & & $x$ & $x$ & $x$ & $x$ & \\
\hline Cytheridea neapolitana Kollmann, 1960 & $x$ & $x$ & $\mathrm{X}$ & $X$ & $X$ & $\mathrm{X}$ & $x$ & $x$ & $X$ & $x$ & $x$ & $\mathrm{X}$ \\
\hline Cytherois uffenordei Ruggieri, 1975 & $x$ & & & & & & & & & & & \\
\hline Cytherois sp. & & & $x$ & & & & & $x$ & $x$ & & & \\
\hline Cytheroma variabilis G. W. Müller, 1894 & & & & & & & & $x$ & & & & $x$ \\
\hline Cytheromorpha fuscata (Brady, 1869) & & & $x$ & & & $x$ & $x$ & & & & & \\
\hline Hemicytherura defiorei Ruggieri, 1953 & $x$ & & $x$ & $x$ & & $x$ & & & & & $x$ & $\mathrm{X}$ \\
\hline Hemicytherura cf. videns (G. W. Müller, 1894) & & $x$ & & & & & & & & & & \\
\hline Hiltermannicythere turbida (G. W. Müller, 1894) & $x$ & $x$ & $x$ & $x$ & $x$ & $x$ & $\mathrm{x}$ & $\mathrm{X}$ & $x$ & $x$ & $x$ & $\mathrm{X}$ \\
\hline Hirschmannia sp. & & & & & $x$ & & & & & & & \\
\hline \multicolumn{13}{|l|}{ Leptocythere bacescoi (Rome, 1942) } \\
\hline Leptocythere istriana Kempf, 2011 & & & & & & $?$ & & & & & & \\
\hline Leptocythere ramosa (Rome, 1942) & & & & $x$ & & & & & & & & \\
\hline Leptocythere rara (G. W. Müller, 1894) & $x$ & & & & $x$ & $x$ & & & & & $x$ & $x$ \\
\hline Leptocythere cf. rara (G. W. Müller, 1894) & $x$ & & $x$ & & & & & & & & & \\
\hline Leptocythere sp. & $x$ & & & & & & & & & & & \\
\hline Loxoconcha affinis (Brady, 1866) & & & & $x$ & $\mathrm{X}$ & $x$ & $\mathrm{X}$ & $x$ & $x$ & $x$ & $x$ & $\mathrm{X}$ \\
\hline Loxoconcha gibberosa Terquem, 1878 & & & & & & & & $x$ & & & & \\
\hline Loxoconcha ovulata (O. G. Costa, 1853) & $x$ & $x$ & $x$ & $X$ & $x$ & $x$ & $x$ & $X$ & $x$ & $x$ & $x$ & $x$ \\
\hline Loxoconcha rhomboidea (Fischer, 1855) & $x$ & $x$ & $x$ & & & & & & & & & \\
\hline
\end{tabular}




\begin{tabular}{|c|c|c|c|c|c|c|c|c|c|c|c|c|}
\hline Loxoconcha stellifera G. W. Müller, 1894 & $\mathrm{X}$ & $x$ & $x$ & & $\mathrm{X}$ & $x$ & & & & & & \\
\hline Microcythere obliqua G. W. Müller, 1894 & & & & & & & & $x$ & & & & \\
\hline Neocytherideis foveolata (Brady, 1870) & $x$ & & & & & & & & & & & \\
\hline Paracytheridea sp. A Uffenorde, 1972 & $x$ & & & & $x$ & & $x$ & & & & & \\
\hline Paracytheridea triquetra (Reuss, 1850) & $\mathrm{X}$ & & & $x$ & $\mathrm{X}$ & $x$ & $x$ & $x$ & $x$ & $x$ & $\mathrm{X}$ & $x$ \\
\hline Paradoxostoma acuminatum G. W. Müller, 1894 & & & & & & & $x$ & & & & & \\
\hline Paradoxostoma triste G. W. Müller, 1894 & & & & & & & & $x$ & $x$ & & & \\
\hline Phlyctocythere sp. & & & $x$ & $\mathrm{x}$ & & & $x$ & & & & & \\
\hline Pontocypris sp. & $x$ & & & & $x$ & & & & & & $\mathrm{X}$ & \\
\hline Pontocythere turbida (G.W. Müller, 1894) & $x$ & $x$ & $\mathrm{x}$ & $\mathrm{x}$ & $x$ & $x$ & $x$ & $x$ & $x$ & $x$ & $x$ & $x$ \\
\hline $\begin{array}{l}\text { Propontocypris (Propontocypris) intermedia } \\
\text { (Brady, 1868) }\end{array}$ & & $x$ & & & & & & & $x$ & & & \\
\hline Pseudopsammocythere similis (G. W. Müller, 1894) & & & & & $x$ & & & & & & & \\
\hline Pterygocythereis jonesii (Baird, 1850) & $\mathrm{X}$ & $x$ & $\mathrm{X}$ & $\mathrm{x}$ & $x$ & $x$ & $\mathrm{x}$ & $x$ & $x$ & $\mathrm{X}$ & $X$ & $x$ \\
\hline Sahnia fasciata (Brady \& Robertson, 1874) & & & & $x$ & $x$ & & $x$ & & $x$ & & $\mathrm{x}$ & \\
\hline Semicytherura acuta (G. W. Müller, 1912) & $x$ & $x$ & & & & & & & & & & \\
\hline $\begin{array}{l}\text { Semicytherura acuticostata ventricosa } \\
\text { (G. W. Müller, 1894) }\end{array}$ & & & & $\mathrm{x}$ & & & & $x$ & & & & \\
\hline Semicytherura alifera Ruggieri, 1959 & & & $x$ & $x$ & $x$ & $x$ & $X$ & & $X$ & & & $x$ \\
\hline Semicytherura diafora Barbeito-Gonzalez, 1971 & & $x$ & & $\mathrm{x}$ & $x$ & $x$ & $x$ & & & & $X$ & $x$ \\
\hline Semicytherura dispar (G. W. Müller, 1894) & & & & $\mathrm{x}$ & & & & & & & & \\
\hline Semicytherura incongruens (G. W. Müller, 1894) & $x$ & $x$ & $\mathrm{X}$ & $\mathrm{X}$ & $X$ & $x$ & $\mathrm{X}$ & $x$ & $x$ & $x$ & $x$ & $x$ \\
\hline Semicytherura inversa (Seguenza, 1880) & & & & & & & & $x$ & $x$ & & $X$ & $x$ \\
\hline Semicytherura quadridentata (Hartmann, 1953) & & & & & & & & & $x$ & & & \\
\hline Semicytherura rara (G. W. Müller, 1894) & & $x$ & $x$ & $x$ & & $x$ & & $x$ & $x$ & & $\mathrm{x}$ & \\
\hline $\begin{array}{l}\text { Semicytherura rarecostata Bonaduce, Ciampo } \\
\text { \& Masoli, } 1976 \text { s.l. }\end{array}$ & $x$ & & $x$ & $x$ & $x$ & $x$ & $x$ & $\mathrm{X}$ & $\mathrm{X}$ & & $\mathrm{X}$ & $x$ \\
\hline Semicytherura sulcata (G. W. Müller, 1894) & $x$ & & & & $x$ & & & & & & & \\
\hline Semicytherura cf. tergestina (cf. Uffenorde, 1972) & & & & & & & $x$ & & $x$ & & $X$ & $x$ \\
\hline Xestoleberis communis G. W. Müller, 1894 & & $x$ & $x$ & $\mathrm{x}$ & $\mathrm{x}$ & $x$ & $\mathrm{x}$ & $\mathrm{x}$ & $x$ & $\mathrm{X}$ & $\mathrm{X}$ & $x$ \\
\hline Xestoleberis spp. & $x$ & $x$ & $\mathrm{x}$ & $\mathrm{X}$ & $x$ & $x$ & $\mathrm{x}$ & $x$ & $x$ & & $x$ & $x$ \\
\hline${ }^{14} \mathrm{C}$ dating (Fütterer \& Paul, 1976) & & & & & & & & & $\begin{array}{l}18 \mathrm{~cm} \\
\text { surfa } \\
6640\end{array}$ & & & \\
\hline Pollen analysis (Beug, 1977) & \multicolumn{9}{|c|}{$\begin{array}{l}\text { Quercus robur dominant; Corylus high } \\
\text { values at the base: reducing/Picea \& Abies } \\
\text { increasing towards core no. 16; Fagus \& } \\
\text { Alnus rel. frequent; Hedera continuously } \\
\text { present; Ulmus strongly varying }\end{array}$} & \multicolumn{3}{|c|}{$\begin{array}{l}\text { cores no. } 17, \\
19 \& \text { surface } \\
\text { sample: no } \\
\text { analysis } \\
\text { possible }\end{array}$} \\
\hline Interpretation (acc. Schmidt et al., 2001) & \multicolumn{9}{|c|}{ Pollen Zone/Subzone: 5/2 } & & & \\
\hline Period & \multicolumn{9}{|c|}{ Atlantic } & & & \\
\hline
\end{tabular}


Shallow marine species, present in the majority of samples, are:

Aurila convexa, Callistocythere adriatica, Carinocythereis whitei, Cytheridea neapolitana, Hiltermannicythere turbida, Loxoconcha affinis, Loxoconcha ovulata, Paracytheridea triquetra, Pontocythere turbida, Pterygocythereis jonesii, Semicytherura alifera, S. incongruens and S. rarecostata as well as xestoleberidids.

This rich, homogeneous ostracod palaeothanatocoenosis is certainly older than the shell layer of unit $\mathrm{C}$ with an oyster shell indicating a ${ }^{14} \mathrm{C}$ age of $6640 \pm 120$ a BP, which can be assigned to the Atlantic period or the Holocene climatic optimum.

In connection with a sea-level curve for the northern Adriatic Sea elaborated by LAMвЕск et al. (2004), the range of the calculated sea-level at $6.6 \mathrm{ka} \mathrm{BP}$ is about -4 to -8 meters below the present sea-level just at the end of the extreme postglacial sea-level rise.

It is remarkable that this ${ }^{14} \mathrm{C}$ age is just at the breaking point between a rapid sea-level rise of $90-100 \mathrm{~cm} / 100$ years (represented by the clayey and sandy silt in units A and B of core PO-1) and a much slower rise of $9-14 \mathrm{~cm} / 100$ years in the fine sand in unit D.

The sediments in the units $C$ and D are interpreted as lag deposits, typical of the sediment-starved North Adriatic shelf (Corregiari et al., 1996). The Highstand Systems Tract (HST) in the last $5 \mathrm{ka}$ BP especially yields more phases of non-deposition than deposition and these latter are concentrated in the western part of the Adriatic Sea (Po delta and adjacent lobes Corregiari et al., 2005).

\section{Ostracoda from core A18 SW of Rovinj/Istria}

The core A18 SW of Rovinj/Istria (Fütterer, 1969; Fütterer \& PAul, 1976) (position: $45^{\circ} 0^{\prime} 49^{\prime \prime} \mathrm{N}, 13^{\circ} 33^{\prime} 23^{\prime \prime} \mathrm{E}$, water depth: $34 \mathrm{~m}$; Fig. 1) has an overall ostracod assemblage similar to that found in core PO-1 (Tab. 3, samples between 5 and $50 \mathrm{~cm}$ ).

The core sample from the uppermost $5 \mathrm{~cm}$ shows an impoverished fauna with smooth Cyprideis torosa as well as its nodose morphotypes, Leptocythere bacescoi, Loxoconcha stelligera and Semicytherura sulcata. This fauna also indicates palaeothanatocoenosis II in a nearshore, even more brackish environment. Although there was no special comment by FütTerer \& PAul (1976), it is assumed that this break in the ostracod record was caused by a burrow bioturbation bringing material from strata deeper than those cored near to the surface.

\section{Ostracoda from a grab sample from position A18 SW of Rovinj/Istria}

In contrast to this result, the uppermost $3 \mathrm{~cm}$ from the same station (position: $45^{\circ} 0^{\prime} 49^{\prime \prime} \mathrm{N}, 13^{\circ} 33^{\prime} 23^{\prime \prime} \mathrm{E}$, water depth: $34 \mathrm{~m}$ ), taken by a Van Veen grab, display a quite different faunal spectrum (Tab. 4). The rich and diverse ostracod assemblage contains Cytherois frequens as part of the biocoenosis (with soft parts and stained with rose bengal). In addition to further specimens of this species without soft parts, Paracytherois flexuosa, Paradostoma simile, Pontocypris acuminata, Xestoleberis communis and other xestoleberidids are part of the thanatocoensosis. All these taxa are thin-shelled. Moreover, the sample is characterized by abundant stained Bryozoa. Also remarkable is the absence of Carinocythereis whitei, Cytheretta subradiosa, Hemicytherura defiorei, Hiltermannicythere turbida, Leptocythere rara, Pontocythere turbida, Pterygocythereis jonesii, and Semicytherura incongruens, S. inversa and S. rarecostata s. 1 .

Although no further investigations were undertaken by FütTERER \& PAUL (1976) or by the author, the recent fauna seems to belong to the "Biocoenosis of detritic bottoms of the open Adriatic Sea" (GAmulin-BridA, 1974, UNEP/MAP, 2015). 
Tab. 3. Ostracoda from core A18, SW of Rovinj/Istria. Light grey arrows towards left: shallowmarine species. Dark grey arrows towards right and rectangles: brackish-lagoonal species.

\begin{tabular}{|c|c|c|c|c|c|c|c|c|c|c|}
\hline $\begin{array}{l}\text { Core A18 } \\
\text { Core No. }\end{array}$ & 10 & 9 & 8 & 7 & 6 & 5 & 4 & 3 & 2 & 1 \\
\hline Core depth $[\mathrm{cm}]$ & $\begin{array}{l}\text { 草 } \\
\text { 占 }\end{array}$ & 隽 & $\begin{array}{l}\text { † } \\
\text { in } \\
\text { m }\end{array}$ & $\begin{array}{l}\text { Ln } \\
\text { on } \\
0\end{array}$ & $\begin{array}{l}\infty \\
\text { ì } \\
\text { ஸे }\end{array}$ & $\begin{array}{l}\stackrel{2}{T} \\
\stackrel{\sim}{N}\end{array}$ & 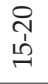 & $\stackrel{20}{\stackrel{2}{2}}$ & $\begin{array}{l}\text { 온 } \\
\text { 它 }\end{array}$ & $\begin{array}{l}20 \\
\vdots \\
\vdots \\
8\end{array}$ \\
\hline \multicolumn{11}{|l|}{ Ostracod taxa } \\
\hline Aurila convexa (Baird, 1850) & & & $x$ & & $x$ & & $x$ & & $\mathrm{X}$ & $X$ \\
\hline Aurila ithacae Uliczny, 1969 & $x$ & & $x$ & & $x$ & & $x$ & & $x$ & \\
\hline Callistocythere adriatica Masoli, 1968 & $\mathrm{X}$ & & $x$ & & $x$ & & $\mathrm{X}$ & & $\mathrm{X}$ & \\
\hline Carinocythereis princeps (Terquem, 1878) & $X$ & & & & & & & & & \\
\hline Carinocythereis whitei (Baird, 1850) & & & $x$ & & & & $x$ & & $\mathrm{X}$ & \\
\hline Cyprideis torosa (Jones, 1850) & $X$ & & $x$ & & $x$ & & $x$ & & $\mathrm{X}$ & $\mathrm{x}$ \\
\hline \multicolumn{11}{|l|}{$\begin{array}{l}\text { Cyprideis torosa (Jones, 1850), nodose } \\
\text { morphotype }\end{array}$} \\
\hline Cytheretta subradiosa (Roemer, 1838) & $x$ & & $x$ & & $x$ & & $x$ & & $\mathrm{X}$ & \\
\hline Cytheridea neapolitana Kollmann, 1960 & & & & & & & & & & $\mathrm{X}$ \\
\hline Cytherois uffenordei Ruggieri, 1975 & & & & & & & & & & $x$ \\
\hline Cytheroma sp. & & & & & $x$ & & & & & \\
\hline Hemicytherura defiorei Ruggieri, 1953 & $X$ & & $\mathrm{X}$ & & $\mathrm{X}$ & & $\mathrm{X}$ & & $\mathrm{X}$ & \\
\hline Hiltermannicythere turbida (G. W. Müller, 1894) & & & $x$ & & $\mathrm{x}$ & & $x$ & & $\mathrm{X}$ & $\mathrm{X}$ \\
\hline \multicolumn{11}{|l|}{ Leptocythere bacescoi (Rome, 1942) } \\
\hline Leptocythere cf. rara (G. W. Mueller, 1894) & $x$ & & & & & & & & & \\
\hline Leptocythere lagunae Hartmann, 1958 (juv.) & $\mathrm{X}$ & & & & & & & & & \\
\hline Leptocythere ramosa (Rome, 1942) & & & & & & & & & $\mathrm{X}$ & \\
\hline Leptocythere rara (G. W. Mueller, 1894) & & & $\mathrm{X}$ & & $\mathrm{X}$ & & & & $\mathrm{X}$ & $\mathrm{X}$ \\
\hline Loxoconcha ovulata (O. G. Costa, 1853) & $x$ & & $\mathrm{X}$ & & $x$ & & & & $\mathrm{X}$ & \\
\hline Loxoconcha rhomboidea (Fischer, 1855) & $x$ & & $x$ & & $x$ & & $x$ & & $\mathrm{X}$ & \\
\hline \multicolumn{11}{|l|}{ Loxoconcha stellifera G. W. Müller, 1894} \\
\hline Microcytherura angulosa (Seguenza, 1880) & $x$ & & & & $x$ & & & & & \\
\hline Neocytherideis foveolata (Brady, 1870) & $x$ & & & & & & & & & \\
\hline Paracytheridea sp. A Uffenorde, 1972 & $X$ & & $\mathrm{X}$ & & $\mathrm{X}$ & & & & & \\
\hline Paracytheridea triquetra (Reuss, 1850) & $X$ & & & & & & $x$ & & $\mathrm{X}$ & \\
\hline Pontocypris sp. & & & & & & & & & & $x$ \\
\hline Pontocythere turbida (G.W. Müller, 1894) & $x$ & & $x$ & & $x$ & & $x$ & & $\mathrm{X}$ & \\
\hline Pseudocythere calcarata (Seguenza, 1880) & & & $\mathrm{X}$ & & & & $x$ & & $\mathrm{X}$ & \\
\hline Pterygocythereis jonesii (Baird, 1850) & $x$ & & $x$ & & $?$ & & $x$ & & $\mathrm{x}$ & \\
\hline Semicytherura acuta (G. W. Müller, 1912) & $x$ & & & & & & $x$ & & $\mathrm{X}$ & \\
\hline $\begin{array}{l}\text { Semicytherura acuticostata ventricosa (G. W. } \\
\text { Müller, 1894) }\end{array}$ & & & $x$ & & & & & & $x$ & \\
\hline
\end{tabular}




\begin{tabular}{|l|l|l|l|l|l|l|l|l|c|c|}
\hline Semicytherura cf. tergestina (cf. Uffenorde, 1972) & & & & & & & $\mathrm{X}$ & & $\mathrm{X}$ & \\
\hline Semicytherura diafora Barbeito-Gonzalez, 1971 & & & & & $\mathrm{X}$ & & & & & \\
\hline Semicytherura incongruens (G. W. Müller, 1894) & $\mathrm{X}$ & & $\mathrm{X}$ & & $\mathrm{X}$ & & $\mathrm{X}$ & & $\mathrm{X}$ & \\
\hline Semicytherura inversa (Seguenza, 1880) & $\mathrm{X}$ & & $\mathrm{X}$ & & $\mathrm{X}$ & & $\mathrm{X}$ & & $\mathrm{X}$ & \\
\hline Semicytherura rara (G. W. Mueller, 1894) & & & & & & & $\mathrm{X}$ & & $\mathrm{X}$ & \\
\hline $\begin{array}{l}\text { Semicytherura rarecostata Bonaduce, Ciampo } \\
\text { \& Masoli, 1976 s.l. }\end{array}$ & $\mathrm{X}$ & & $\mathrm{X}$ & & $\mathrm{X}$ & & $\mathrm{X}$ & & $\mathrm{X}$ & $\mathrm{X}$ \\
\hline Semicytherura sulcata (G. W. Müller, 1894) & & & & & & & & & & $\mathrm{X}$ \\
\hline Xestoleberis communis G. W. Müller, 1894 & & & $\mathrm{X}$ & & $\mathrm{X}$ & & $\mathrm{X}$ & & $\mathrm{X}$ & \\
\hline Xestoleberis spp. & $\mathrm{X}$ & & $\mathrm{X}$ & & $\mathrm{X}$ & & $\mathrm{X}$ & & $\mathrm{X}$ & \\
\hline
\end{tabular}

However, a further component could be proven for the first time in sediments of offshore Istria. This is a group of species indicating warmer water. Four species can be included in this group: Cytherelloidea beckmanni, Triebelina raripila, Aurila interpretis and Hemicytherura gracilicosta. These are representatives of the palaeothanatocoenosis I in the sense of BREMAN (1976) with species which come from a southern climatic province via the northwards, anti-clockwise directed East Adriatic Currents (EAC, UNEP/MAP, 2015). Cytherelloidea beckmanni has been proven as strong climatological indicator (BARRA, 1997). Melis \& Pugliese (1985) found Cytherelloidea beckmanni together with Hemicytherura gracilicosta in association with Posidonia oceanica (? Meadows or banks of dead leaves of Posidonia oceanica) west of Sicily. Bonaduce et al. (1976) interpreted Aurila interpretis as characteristic of the Levant Basin. They found this species only once frequently (SSE Italy between Lecce and San Maria di Leuca, near-shore by diving). The frequent occurrence of Aurila interpretis at a single station E of Venice, reported by BREMAN (1976, tab. III d), seems to be due to reworking (so-called Pleistocene age sediment). BARRA (1997) mentioned Triebelina raripila as a species that prefers the southern part of the Mediterranean Sea, but may have scattered occurrences further north. This occurrence is here interpreted as influenced by currents like the EAC mentioned above.

\section{DISCUSSION}

As exemplified by the two cores PO-1 and A18 drilled offshore from the Istrian Peninsula (Croatia), the northern Adriatic Sea bottom shows a very complicated composition connected with the postglacial sedimentation and re-deposition. The denudation of sediments deposited in the late glacial/postglacial Transgressive Systems Tract, lag deposition during the Maximum Flooding Surface and the Highstand Systems Tract play an important role in the actual sediment pattern. It has to be borne in mind that many of the shells found at the Adriatic Sea bottom are in fact fossils. This could be proven in the present study by ostracods.

The very sandy pelite community (stations $2-4$ ) and the fine sand community (station 5) described by BonADUCE et al. (1976) are not genuine recent or subrecent death assemblages. They are predominantly palaeothanatocoenoses with different palaeoenvironmental depth zones due to the postglacial sea-level change (palaeothanatocoenoses II of BREMAN (1976)). The widespread Pleistocene of the northern Adriatic Sea postulated by Breman (1976) could not be reached, either in the stations sampled by BonADUCE et al. (1976), or by FütTERER \& PAUl (1976) or the present study. 
Tab. 4. Ostracoda from a Van Veen grab sample from position A18, SW of Rovinj/Istria.

\begin{tabular}{|l|l|}
\hline Station A18 (Van Veen grab) [cm below surface] & 00-ca.03 \\
\hline Ostracod taxa & \\
\hline Aurila convexa (Baird, 1850) & $\mathrm{X}$ \\
\hline Aurila interpretis Uliczny, 1969 & $\mathrm{X}$ \\
\hline Aurila ithacae Uliczny, 1969 & $\mathrm{X}$ \\
\hline Bairdia spp. & $\mathrm{X}$ \\
\hline Callistocythere adriatica Masoli, 1968 & $\mathrm{X}$ \\
\hline Callistocythere littoralis (G. W. Mueller, 1894) & $\mathrm{X}$ \\
\hline Callistocythere lobiancoi (G. W. Mueller, 1894) & $\mathrm{X}$ \\
\hline Cytherelloidea beckmanni Barbeito-Gonzalez, 1971 & $\mathrm{X}$ \\
\hline Cytherois frequens G. W. Mueller, 1894 & $\mathrm{X}$ \\
\hline Hemicytherura gracilicosta (Ruggieri, 1953) & $\mathrm{X}$ \\
\hline Jugosocythereis prava (Baird, 1850) & $\mathrm{X}$ \\
\hline Loxoconcha affinis (Brady, 1866) & $\mathrm{X}$ \\
\hline Loxoconcha ovulata (O. G. Costa, 1853) & $\mathrm{X}$ \\
\hline Loxoconcha rhomboidea (Fischer, 1855) & $\mathrm{X}$ \\
\hline Microcytherura angulosa (Seguenza, 1880) & $\mathrm{X}$ \\
\hline Neonesidea longevaginata (G. W. Mueller, 1894) & $\mathrm{X}$ \\
\hline Paracytheridea sp. A Uffenorde, 1972 & $\mathrm{X}$ \\
\hline Paracytheridea triquetra (Reuss, 1850) & $\mathrm{X}$ \\
\hline Paracytherois flexuosa (Brady, 1867) & $\mathrm{X}$ \\
\hline Paradoxostoma simile G. W. Mueller, 1894 & $\mathrm{X}$ \\
\hline Pontocypris acuminata G. W. Müller, 1894 & $\mathrm{X}$ \\
\hline Semicytherura acuticostata ventricosa (G. W. Mueller, 1894) & $\mathrm{X}$ \\
\hline Semicytherura alifera Ruggieri, 1959 & $\mathrm{X}$ \\
\hline Triebelina raripila (G. W. Mueller, 1894) & $\mathrm{X}$ \\
\hline Urocythereis sp. juv. & $\mathrm{X}$ \\
\hline Xestoleberis communis G. W. Mueller, 1894 & $\mathrm{X}$ \\
\hline Xestoleberis spp. & \\
\hline
\end{tabular}

Many of the new species created by Bonaduce et al. (1976) are not actually living. Their inclusion into zoological databases like World Register of Marine Species (WoRMS), World Ostracoda Database, Encyclopedia of Life (eol), European Register of Marine Species (ERMS) and other zoological databases should be marked as Quaternary.

Quite a number of northern Adriatic Sea Ostracoda have been attributed to recent habitats - based only on the study of empty valves or carapaces. In view of sedimentological studies which can be traced back to van StraAten (1970) and Fütterer \& Paul (1976) this assumption has to be questioned.

Information is given here on the Holocene lagoonal to marine ostracod succession from the cores PO-1, SW of Poreč and A18, SW of Rovinj (both of offshore Istria, Croatia), 
which was taken by FüTTERER (1969). Both cores show a relatively uniform ostracod assemblage $5 \mathrm{~cm}$ and deeper below sediment surface, which clearly indicates a death assemblage from a different palaeoenvironmental depth zone due to the postglacial sea-level change (palaeothanatocoenosis II). In the uppermost $3-5 \mathrm{~cm}$, the ostracod composition is extremely different from sample to sample, depending on bioturbation, storm re-deposition or lag deposition.

At station A18, storm deposits (abundance of Bryozoa) with a mixture of biocoenotic, thanatocoenotic and palaeothanatocoenotic ostracods were recorded for the first time in the northeastern Adriatic Sea. The latter group of ostracods belongs to a death assemblage derived from the southern Adriatic or other parts of the Mediterranean Sea (palaeothanatocoenosis I).

Taking samples for 'living' Ostracoda requires the use of appropriate sampling devices like high momentum gravity corer and Krumm bottom sampler, the optimum in gathering of a sediment sample without, or with as little as possible, turbulence on the bottom, retrieving the grab sampler with the least possible undulation of the sea and ladling the surface of the sample onboard.

Although only a few studies have dealt with the eastern part of the Northern Adriatic Sea, it is clear that wide areas of the sea floor are covered generally by reworked lag deposits, which were created in connection with the Early Holocene marine transgressions (Transgressive Systems Tract about 9-7 ka BP) and the Holocene climatic optimum (Maximum Flooding Surface/Condensed Interval about 6-5 ka BP, Corregiari et al., 2005). The transgressive surface beneath these lag deposits seems to be much older, probably 12-14 ka BP (Dinelli et al. (2012).

The attribution of 46 new species, erected by BonADuce et al.(1976), to the recent fauna of the Adriatic Sea in the "World Register of Marine Species" (WoRMS)/ "World Ostracoda Database", "Encyclopedia of Life" (eol), North Atlantic Register for Marine Species (NARMS), "European Register of Marine Species" (ERMS)/MarBEF Data System" (marbef) and other zoological databases should - at least partially - be used with caution.

Moreover, no information concerning the position of the type localities of these new taxa was given by BonAduce et al. (1976). For the first time, latitude/longitude data can be reconstructed and presented here (with the help of the OGA National Oceanographic Data Centre, Trieste).

It is proposed that the inclusion of these taxa into general zoological data bases should be marked as Quaternary in order to distinguish them from living ostracods.

\section{ACKNOWLEDGEMENTS}

Special thanks are due to M. Vinci (Istituto Nazionale di Oceanografia e di Geofisica Sperimentale - OGS Dipartimento di Oceanografia - OGA National Oceanographic Data Centre, Trieste) for his support with the oceanographic data of the Cruise ADRIATIC 1965-66 (analog data given by L. Trotti, 1969). A. R. Lord (Frankfurt/London) is thankfully acknowledged for reviewing the manuscript and improving the English, and an anonymous Italian reviewer is thanked for helpful suggestions and comments.

The author is also indebted to D. Fütterer (Bremerhaven) and J. Paul (Göttingen) for the excellence in preparation and keeping of the sediment samples from the cores $\mathrm{PO}-1$ and A18 (recovery by the author in 2004 after flooding of the storage-cellar), which could easily be re-studied micropalaeontologically. 


\section{REFERENCES}

Aiello, G. \& Barra, D., 2010: Crustacea Ostracoda. Biologia Marina Mediterranea 17 (suppl. 1), 401-419.

Aiello, G., Barra, D. \& Bonaduce, G., 1995: Crustacea Ostracoda. Checklist delle Specie della Fauna Italiana 27. Calderini: Bologna. ISBN 88-7019-973-8. 19 pp.

Ascoli. P., 1965: Crociera talassografica Adriatica 1955. VI. Ricerche ecologiche sugli ostracodi contenuti in 16 carote prelevata sul fondo del Mare adriatico. Archivio di Oceanografia e Limnologia 14 (1), 69-137.

BARRA, D., 1997: The shallow-water ostracods of Tripoli (Libya) and their geographical distribution in the Mediterranean. Revista Española de Micropaleontologia 29 (3), 71-106.

BEug, H.-J., 1977: Vegetationsgeschichtliche Untersuchungen im Küstenbereich von Istrien (Jugoslawien). Flora 166, 357-381.

Breman, E., 1976: The distribution of ostracodes in the bottom sediments of the Adriatic Sea. Vrije Universiteit te Amsterdam, Acad. Proefschrift. Krips Repro, Meppel. 1-165, I-XX, A1-A19.

Bonaduce, G., Ciampo, G. \& Masoli, M., 1976: Distribution of Ostracoda in the Adriatic Sea. Pubblicazioni della Stazione Zoologica di Napoli 40 (Supplement for 1975), 1-304.

Bonaduce, G., Montenegro, M. E. \& Pugliese, N., 2004: The brackish water ostracod Cyprideis torosa (Jones) in marine deposits: the case of the Adriatic Sea. Bollettino della Società Paleontologica Italiana 43 (1-2), 267-271.

Ciliberto, B. M. \& Pugliese, N., 1980: Ostracodi bentonici del tratto di mare compreso tra Grado e Caorla (Adriatico settentrionale). Gortania - Atti del Museo Friulano di Storia Naturale 2, 65-80.

Corregiari, A., Field, M. E. \& Trincardi, F., 1996: Later Quaternary transgressive large dunes on the sediment-starved Adriatic shelf. In: De Batist, M. \& Jacobs, P. (eds.) Geology of siliciclastic shelf seas. Geological Society Special Publication 117, 155-169.

Corregiari, A., Cattaneo, A. \& Trincardi, F., 2005: The modern Po Delta system: Lobe switching and asymmetric prodelta growth. Marine Geology 222-223: 49-74.

Dinelli, E., Ghosh, A., Rossi, V. \& Vaiani, S. C., (2012): Multiproxy reconstruction of Late PleistoceneHolocene environmental changes in coastal successions: microfossil and geochemical evidences from the Po Plain (Northern Italy). Stratigraphy 9 (2), 153-167.

Fütterer, D., 1969: Die Sedimente der nördlichen Adria vor der Küste Istriens. Göttinger Arbeiten zur Geologie und Paläontologie 3, 1-57.

Fütterer, D. \& Paul, J., 1976: Recent and Pleistocene sediments off the Istrian Coast (Northern Adriatic, Yugoslavia). Senckenbergiana maritima 8 (1/3), 1-21.

Gamulin-Brida, H., 1974: Biocoenoses benthiques de la mer Adriatique. Acta Adriatica 15 (9), 1-102.

Kемpf, E. K., 2011: Juxilyocypris gen. nov. and replacement names for homonym species or genera of Ostracoda (Arthropoda: Crustacea). Munis Entomology \& Zoology 6 (2), 955-969.

Klöppel, A., Messal, C., Pfannkuchen, M., Matschullat, J., Zucht, W., Hamer, B. \& Brümmer, F., 2011 : Abiotic sponge ecology conditions, Limski Kanal and Northern Adriatic Sea, Croatia. Open Journal of Marine Science 1, 18-30.

Lambeck, K., Antonioli, F., Purcell, A. \& Silenzi, S., 2004: Sea-level change along the Italian coast for the past 10,000 yr. Quaternary Science Reviews 23, 1567-1598.

Masoli, M., 1968: Ostracodi recenti dell'Adriatico settentrionale tra Venezia e Trieste. Memorie del Museo Tridentino di Scienze Naturali 17 (1), 1-100.

Masoli, M., 1969: Distribution of species of the genus Semicytherura in the Northern Adriatic Sea between Venice and Trieste, Italy. In: NeALe, J. W. (ed.), The taxonomy, morphology and ecology of Recent Ostracoda. Oliver \& Boyd Edinburgh, 334-354.

Meischner, D., 1973: Formation processes and dispersal patterns of the sediments along the Istrian coast of the Adriatic. Rapport Commission International Mer Mediterranee 21 (11): 843-846.

Meischner, D. \& Rumohr, J., 1974: A light-weight, high-momentum gravity corer for subaqueous sediments. Senckenbergiana maritima 6, 105-117.

Melis, R. \& Pugliese, N., 1985: Le Ostracofaune bentoniche delle Isole Egadi (Sicilia). Atti del Museo Geologico e Paleontologico di Monfalcone 1985 (3), 1-22.

Montenegro, M. E., Pugliese, N. \& Bonaduce, G., 1998: Shelf ostracods distribution in the Italian seas. In: Crasquin-Soleau, S., Braccini, E. \& Lethiers, F. (eds.), What about Ostracoda! Actes du 3e Congrès Européen des Ostracodologistes, 1996. Bulletin du Centre Recherche Elf Exploration Production, Mémoire 20, 91-101. 
PAUL, J., 1970: Sedimentgeologische Untersuchungen im LIM und vor der istrischen Küste (nördliche Adria). Göttinger Arbeiten zur Geologie und Paläontologie 7, 1-75.

Rossi,V., 2009: Ostracod assemblages from Holocene subsurface deposits of modern Po Delta: a palaeoenvironmental proxy record. Bollettino della Società Paleontologica Italiana 48 (2), 95-103.

Schmidt, R. Pugliese, N., Müller, J., Szeroczyriska, K., Bogner, D., Melis, R., Kamenik, C., Barić, D \& Danielopol, D. L., 2001: Palaeoclimate, vegetation and coastal lake development, from upper pleniglacial until early Holocene, in the northern Adriatic Valum Bay (Isle of Cres, Croatia). Il Quaternario $14(1), 61-78$.

SокAČ, A., 1975: Mikrofauna sedimenata morskog dna iz Crnogorskog Primorja. Studia Marina 8, 111120.

Sokač, A. \& Hajek-Tadesse, V., 1993: Ostracode fauna of the Adriatic Sea. In: Mckenzie, K. G. \& Jones, P. J. (eds.), Ostracoda in the earth and life sciences. Proceedings of the $11^{\text {th }}$ international Symposium on Ostracoda, Warrnambool, Victoria, Australia, 8-12 July 1991, A. A. Balkema Rotterdam, 515-528.

Trotтr, L., 1969: Crociere Mare Adriatico 1965-1966. CNR, Raccolta dati oceanografici, Serie A, no. 29, 1-82. [partly published under http://nettuno.ogs.trieste.it/medar/IO48.html, Cruise Ref. IO48196565001].

UfFENORDE, H., 1972: Ökologie und jahreszeitliche Verteilung rezenter benthonischer Ostracoden des Limski kanal bei Rovinj (nördliche Adria). Göttinger Arbeiten zur Geologie und Paläontologie 13, $1-121$.

UfFENORDE, H., 1975: Dynamics in Recent marine benthonic ostracode assemblages in the Limski kanal (northern Adriatic Sea). In: Swain, F.M. et al. (eds.) Biology and paleobiology of Ostracoda. Symp. Univ. Delaware 1972. Bulletin of American Paleontology 65 (282), 147-165.

UfFenORde, H., 2015: Living and Quaternary Ostracoda from the Eastern Adriatic Sea: Biocoenosis, thanatocoenosis or palaeothanatocoenoses? - In: Perrier, V. \& Meidla, T. (eds.). Abstracts, 8th European Ostracodologists' Meeting. Tartu, Estonia, 22-30 July 2015, 81-82.

UNITED NATIONS ENVIRONMENT PROGRAMME / Mediterranean Action Plan, 2015: Twelfth Meeting of Focal Points for SPAs (Athens, Greece, 25-29 May 2015) Adriatic Sea: Ecology (draft report). UNEP(DEPI)/MED WG.408/Inf.14 [published under http://www.rac-spa.org/nfp12/documents/information/wg.408_inf14_eng.pdf].

van Straaten, L. M. J. U., 1970: Holocene and late-Pleistocene sedimentation in the Adriatic Sea. Geologische Rundschau 60 (1), 106-131.

von Daniels, C. H., 1970: Quantitative ökologische Analyse der zeitlichen und räumlichen Verteilung rezenter Foraminiferen im Limski-kanal bei Rovinj (nördliche Adria). Göttinger Arbeiten zur Geologie und Paläontologie 8, 1-109.

von Daniels, C. H., Meischner, D. \& Uffenorde, H., 1970: Ein verbesserter quantitativer Bodengreifer nach H. Krumm. Meyniana 20, 1-3. 